El primero te lo regalan: zero-rating de las operadoras móviles de la Argentina

Lucas Enzo Lufrano

Question, Vol. 1, N. 62, e163, abril-junio 2019

ISSN 1669-6581 | https://doi.org/10.24215/16696581e163

http://perio.unlp.edu.ar/ojs/index.php/question

FPyCS | Universidad Nacional de La Plata

La Plata | Buenos Aires | Argentina

\title{
El primero te lo regalan: zero-rating de las operadoras móviles de la Argentina
}

The first one's free: zero-rating of mobile networks operators in Argentina

\author{
Lucas Enzo Lufrano lucaslufrano@gmail.com \\ http://orcid.org/0000-0002-4246-1476 \\ Universidad Nacional de Quilmes (Argentina)
}

\section{Resumen}

Se exploran las prácticas de zero-rating o tasa cero implementadas por las operadoras de internet móvil de la Argentina en 2017 y 2018 y la respuesta del ente regulador, desde el enfoque de la Economía Política de la comunicación y la noción de neutralidad de la red, en el 
contexto de concentración convergente y de emergencia de un nuevo paradigma regulatorio en el sector infocomunicacional.

Mediante la consulta de bibliografía, artículos periodísticos, informes del sector y documentos legales, se concluye que no basta un marco normativo que prohíba dichas promociones mientras no se complemente con una autoridad de aplicación independiente del gobierno y de los intereses privados y una política pública de comunicación que se proponga restringir la fuerza centrípeta del mercado.

Palabras clave: Neutralidad de la red; concentración; convergencia; políticas públicas; comunicación.

\section{Abstract}

This paper explores zero-rating practices implemented by mobile network operators in Argentina during 2017 and 2018 and the regulator's response, from the perspective of the Political Economy of Communication and the notion of net neutrality, in the context of converging concentration and the emergence of a new regulatory paradigm in the info communicational sector.

Through the study of bibliography, news articles, sector reports and legal documents, it is concluded that a regulatory framework that prohibits such promotions is not enough without an independent enforcement authority (of the government and of private interests) and a public communication policy that aims to restrict the market's centripetal force.

Keywords: Net neutrality; concentration; convergence; public politics; communication.

El presente artículo explora las prácticas de zero-rating o tasa cero en la Argentina durante 2017 y 2018, así como las respuestas del organismo regulador. Aquéllas consisten en promociones mediante las cuales las prestadoras de internet no descuentan el consumo de ciertos contenidos, servicios o aplicaciones de la cuota de datos contratada. Esta diferenciación tarifaria colisiona con la noción de neutralidad de la red, que impone el trato igualitario sobre todo el tráfico que circula por ella. A pesar de que el marco legal vigente garantiza este principio, las operadoras móviles bonifican a sus clientes el uso de ciertas determinadas funciones de la aplicación de mensajería Whatsapp desde 2016. De manera similar, y en el marco de la fusión entre Telecom y Cablevisión, Personal ofreció el mismo beneficio a una 
parte de sus usuarios para visualizar en vivo eventos deportivos y musicales a través de su servicio de streaming audiovisual Flow.

A partir de la consulta de bibliografía, se precisa el concepto de zero-rating en su relación con el de neutralidad de la red y sus implicancias. Luego, mediante el análisis de informes de la industria, artículos periodísticos, leyes y documentos públicos, se analizan los casos mencionados y las actuaciones del organismo regulador en el contexto de la concentración convergente del sector infocomunicacional y el giro de las políticas para el mismo.

\section{Neutralidad de la red}

Concebida como un servicio para conectar distintas redes de computadoras entre sí, el diseño original de internet responde a criterios de eficiencia comunicativa con el objetivo de garantizar el flujo descentralizado de información. Entre otros elementos, la transferencia y recepción de datos entre sus múltiples puntos requiere el empleo de reglas, conocidas como protocolos (Cortés Castillo, 2016). El TCP/IP - Transmission Control Protocol/Internet Protocol-, el estándar principal de la red, funciona en base a tres principios interdependientes. El primero, la conmutación de paquetes, dispone que la información, indistintamente de su contenido o características, se descomponga en origen y viaje en cualquier orden por rutas distintas hasta su destino, donde es reensamblada. El segundo, de "extremo a extremo", circunscribe la función de la red al mero transporte homogéneo de paquetes entre terminales $\mathrm{Y}$ el tercero la estratifica en capas con funciones separadas, jerarquizadas y encadenadas: desde el nivel físico hasta el de aplicaciones (Cortés Castillo, 2014).

Estas características sustentan técnicamente la noción de neutralidad de la red, que impone el trato igualitario de todo el tráfico de datos, rechazando distorsiones intencionales de los actores intervinientes que discriminen positiva o negativamente determinados contenidos o servicios (Chaparro, 2014; Fontanals, 2016). Su objetivo es evitar que se afecten derechos humanos fundamentales como el acceso a la información y la libertad de expresión.

Sin embargo, en los hechos, el tráfico en la web no viaja de manera homogénea. En principio, la propia arquitectura estipula, mediante el protocolo de "calidad de servicio" que cierto tipo de paquetes se prioricen más allá de su carga puntual de datos (Gendler, 2017). Además, los operadores de red aplican temporalmente medidas de gestión de tráfico para lidiar con situaciones de congestión o peticiones de bloqueo, para lo cual introducen dispositivos y tecnologías para monitorear el flujo de información, quebrando los principios de estratificación y de "extremo a extremo" (Cortés Castillo, 2014). Por último, los proveedores de contenidos de 
mayor tamaño contratan servicios de redes de entrega de contenidos (CDN) o instalan sus propios servidores para favorecer su tráfico. Por todo ello, una parte de la literatura prefiere el término apertura de la red, inclinándose hacia garantizar la libertad de acceso y elección de los usuarios.

La cuestión es emergente de la expansión masiva y mutación de internet tras la privatización de los puntos principales de conexión en 1995 y la consecuente mercantilización del acceso (Cortés Castillo, 2016), así como de las alteraciones en la forma de conexión y en el tipo de contenidos consumidos merced de la interacción entre las novedades tecnológicas y los usos y costumbres de los usuarios. Desde entonces, el tráfico a nivel mundial creció un 100.272.325\% (Sumits, 2015), motorizado por la ascendente penetración del servicio de banda ancha, tanto fija como móvil (Rodríguez Miranda y Carboni, 2012). Además, su composición mutó desde el predominio del correo electrónico y la navegación de páginas web hacia el del streaming audiovisual, el alojamiento de archivos en la nube y la consulta de redes sociales. Entre 2006 y 2016, la participación del video sobre el tráfico global subió del 9 al 73\%, con la proyección de alcanzar el 82\% en 2021 (Cisco, 2018). El incremento del consumo audiovisual online es producto de la convergencia, inicialmente tecnológica, que unifica soportes de producción, edición, distribución y consumo, productos y lógicas de emisión y recepción de medios audiovisuales, gráficos, telecomunicaciones y redes digitales (Becerra, 2015).

Con una internet reconfigurada hacia la valorización del capital, los crecientes ingresos son disputados por los jugadores más pesados del ecosistema digital (Califano y Baladrón, 2012), cuyas posiciones en el campo aún están en pugna. Por un lado, los operadores de red: las telefónicas y las cableras. Por el otro, los proveedores de contenidos, especialmente aquellos intermediarios globales que prestan servicios over-the-top (OTT). Los primeros reprochan a los segundos que hacen un uso intensivo de la red, beneficiándose de su condición de free-riders, sin compartir los gastos y las inversiones necesarias para mantener y expandir la infraestructura (Fontanals, 2016). Netflix y Youtube acaparaban el $26 \%$ de la descarga global de contenidos en 2018 , con aquél alcanzando un 40\% durante las horas pico en el conjunto del continente americano (Sandvine, 2018).

En esta contienda, los operadores de red cuentan con cierto poder para moldear el entorno dado su control sobre la red física y lógica (Pallero, 2018). En efecto, detentan la ventaja de gestionar el tráfico de las terminales, con la posibilidad técnica de inspeccionar los paquetes que circulan sobre la red y alterar el flujo de determinados contenidos o servicios (Cortés Castillo, 2014; Gendler, 2017). Ello les permite, por ejemplo, presionar a los proveedores de contenidos para cobrarles los servicios especializados ya mencionados, a modo de peaje como inversión cruzada (Fontanals, 2016). El problema se agudiza en el contexto de convergencia 
infocomunicacional, también económica, donde la integración vertical entre los medios audiovisuales y las telecomunicaciones genera firmas con dominio sobre las fases de producción y distribución de contenidos y servicios (Becerra, 2003), con la posibilidad de priorizar los suyos o los de un aliado por sobre los de la competencia (Cortés Castillo, 2016; Observacom, 2018).

Como cuestión no tratada por los organismos globales de ordenación técnica de internet (Califano y Baladrón, 2013) y aunque aún no existe un acuerdo generalizado para imponer una regulación por vía reglamentaria, algunos Estados avanzaron sobre dicho desbalance cristalizando la demanda de neutralidad en leyes focalizadas sobre los operadores (Becerra, 2017). Estos no sólo resultan más abordables por su anclaje territorial -contra la deslocalización de los intermediarios digitales-, sino también por su inscripción a un ámbito regulatorio existente: la neutralidad se enraiza en las reglas de acceso abierto y transporte común de las telecomunicaciones (Cortés Castillo, 2014; Fernández, 2014).

\section{Zero-rating}

Donde el Estado efectivamente concretó reglamentaciones, empero, la definición sobre neutralidad no es unívoca. El consenso en prohibir las medidas de bloqueo o degradación no se extiende en todos los casos a las prácticas de priorización. Este último grupo incluye las acciones de zero-rating o tasa cero, una forma de segregación tarifaria vía precios, mediante la cual las operadoras bonifican el consumo de determinados contenidos, servicios 0 aplicaciones, evitando que su tráfico sea deducido de las cuotas de planes de datos (Fontanals, 2017). Sin embargo, la contracara de tal aliento supone la discriminación negativa sobre el resto de los contenidos no incluidos en la promoción o los usuarios no alcanzados por los planes establecidos.

El costo de dicho consumo, aparentemente gratuito, es afrontado por los proveedores de contenidos para promocionar su uso o, generalmente, por las operadoras para apalancar los suyos o para aumentar o conservar su clientela. A su vez, esta práctica comercial es más común entre los proveedores de internet móvil, donde tienden a prevalecer los planes de acceso con tráfico medido con un límite máximo de datos, cuyo exceso demanda un pago adicional del cliente (Intervozes y Derechos Digitales, 2017). A diferencia de la tarifa plana, más asidua en las conexiones fijas, este modelo apunta a trasladar el costo de las inversiones a los usuarios mediante una facturación atada al consumo, arquetipo de financiamiento de las telecomunicaciones (Becerra, 2003). 
Fontanals (2017a) identifica tres modelos de zero-rating: a. un proveedor de contenidos se une a una operadora para otorgar acceso a internet restringido a su plataforma, sin exigir contratar un abono; b. una empresa de telecomunicaciones integrada verticalmente bonifica sus propios servicios; y c. una operadora favorece ciertos servicios ajenos y masivos. En cualquier caso, estos programas distorsionan el consumo de contenidos y el acceso al mercado, actúan en mayor o menor medida, como jardines vallados que restringen la experiencia e incrementan el poder de quienes controlan la red (McSherry, Malcolm y Walsh, 2016). Todo ello beneficia a los actores más poderosos, retroalimentando la concentración del sector infocomunicacional, que ya por su propia lógica tiende a la concentración, y desalienta la competencia y la innovación (Becerra y Mastrini, 2017). Además, las operadoras trasladan las pérdidas de cubrir el zerorating hacia el precio o cuota de los planes. Allá donde dichas prácticas no son aceptadas, los abonos suelen incluir un mayor número de datos (Fontanals, 2017).

\section{Concentración convergente en la Argentina}

De manera congruente con Latinoamérica, el sector infocomunicacional en la Argentina presenta una estructura oligopólica, con presencia de capitales financieros y/o extranjeros y un comportamiento conglomeral que camina hacia la convergencia entre sus dos actividades más lucrativas: las telecomunicaciones y el audiovisual (Mastrini y Becerra, 2018). Si bien en el último cuarto de siglo el dominio recayó sobre dos grandes grupos, Telefónica y Clarín, la relación de fuerzas tiende a modificarse desde 2017 a partir de la unión de Cablevisión -la cable operadora y prestadora de internet fijo que explicaba el $75 \%$ de los ingresos del segundocon Telecom -la telefónica que también pisaba el mercado sin hilos y el de conectividad en sus dos variantes-.

La fusionada, con participación de capitales nacionales, concentra el $42 \%$ de la telefonía fija (Telecom), el 34\% de la móvil (Personal, de Telecom), el 56\% de las conexiones fijas a internet (Fibertel, de Cablevisión, y Arnet, de Telecom), el 35\% de las móviles (Personal y Nextel, de Cablevisión) y el $40 \%$ de los abonos de TV paga (Cablevisión). Su pata en el sistema de medios publica el principal periódico nacional, que a su vez tiene una destacada posición en la web, lidera el espacio radiofónico AM y FM, posee la primera señal de TV de pago y la segunda de TV abierta más vistas, además de participar en la edición de revistas, libros y otros periódicos, en la fabricación de papel de diario y en la producción televisiva y cinematográfica. Así, ostenta posiciones privilegiadas en la producción de contenidos y en su distribución por distintas vías. 
Por su parte, Telefónica, el mayor grupo de la región y con capitales mayoritariamente españoles, detenta el $43 \%$ de la telefonía fija, el 33\% de la móvil (Movistar), el $27 \%$ de los accesos fijos de banda ancha (Speedy) y el 32\% de los móviles (Movistar). En 2016 se desprendió de sus señales de televisión abierta. Mientras ambos holdings conforman un casi duopolio en la telefonía fija -cuya penetración alcanza al $71 \%$ pero se encuentra en franca disminución-, los servicios móviles -133 accesos cada 100 habitantes- se reparten en tercios: Telmex, el segundo actor del subcontinente y de capitales regionales, controla el $36 \%$ de internet y el $29 \%$ de la telefonía mediante su firma Claro. A diferencia de su estrategia general en Latinoamérica, ni Telefónica ni Telmex se expandieron aún hacia la prestación de televisión de pago en el país, que llega al $80 \%$ de los hogares. El otro actor de peso que se posiciona allí es la norteamericana AT\&T mediante DirecTV, la única operadora satelital habilitada en el territorio argentino, que posee el $30 \%$ de los abonos de TV paga.

A grandes rasgos, las políticas públicas acompañaron y alentaron esta estructuración del mercado desde las últimas décadas del siglo pasado y, cuando actuaron en sentido contrario, no lograron revertirla (Becerra, 2015). En una primera fase, durante las décadas de 1980 y 1990, la privatización de los servicios públicos y los cambios normativos en el sistema de medios permitieron, por un lado, el reparto de la telefónica estatal entre los capitales extranjeros de Telefónica y Telecom, que a la postre les serviría de plataforma para expandirse al servicio móvil y a internet, y, por el otro, la conformación de multimedios con transmisión en red como el Grupo Clarín y la misma Telefónica. La crisis económica y social de cambio de siglo justificó una segunda etapa de concentración defensiva. Bajo el pretexto de evitar una invasión de intereses foráneos, Clarín obtuvo luz verde para que su cable operadora Multicanal absorbiera a su principal competidora, Cablevisión, y bloqueó el ingreso de las telefónicas al mercado de cable. A partir de 2008, en línea con el clima regional, se produce un giro en la regulación hacia un mayor intervencionismo estatal con la denuncia de la concentración como un problema. La ruptura entre Clarín y la administración de Cristina Fernández de Kirchner, que se extendió hasta el fin de su segundo mandato, se cristalizó, entre otras medidas, en la Ley de Servicios de Comunicación Audiovisual (2009), que pretendía desconcentrar el sector y evitar su convergencia con las telecomunicaciones. Sin embargo, su aplicación demorada por los litigios judiciales, enfocada únicamente en el conglomerado rival del gobierno y tolerante con el resto resultó ineficaz. Asimismo, su espíritu fue modificado desde fines de 2014 con la sanción de la Ley Argentina Digital, que habilitó legalmente el ingreso de las telefónicas en la televisión por cable, inaugurando una tercera fase de concentración convergente.

Esta última etapa se intensificó a partir del viraje en las políticas de comunicación tras el cambio de gobierno en diciembre de 2015. Con una activa intervención mediante decretos y

Question, Vol. 1, N.0 62, abril-junio 2019. ISSN 1669-6581

Instituto de Investigaciones en Comunicación | Facultad de Periodismo y Comunicación Social | Universidad Nacional de La Plata La Plata | Buenos Aires | Argentina

Página 7 de 15 
resoluciones centralizadas y la nunca concretada promesa de sancionar una nueva ley convergente, la normativa fue adaptada a la dinámica del mercado con una orientación en favor de los actores dominantes e intentado arbitrar entre sus intereses particulares contrapuestos. Además, la nueva gestión no sólo dio por finalizada la contienda con el Grupo Clarín sino que tendió a favorecerlo.

Los límites a la concentración y a la propiedad cruzada fueron eliminados de la Ley Audiovisual, que además perdió injerencia sobre la televisión por cable (Marino, 2018a). Asimismo, su autoridad de aplicación (AFSCA) se unificó con la de la Ley Argentina Digital (AFTIC), concerniente al sector de las telecomunicaciones resultando un organismo aún más gubernamentalizado. El Ente Nacional de Comunicaciones (ENACOM) no sólo reforzó su subordinación institucional al Ejecutivo nacional sino que algunas decisiones directamente fueron delegadas a otros organismos gubernamentales (Fontanals, 2017c).

Posteriormente, habilitó al Grupo Clarín a brindar telefonía móvil, a DirecTV a ofrecer internet y a las telefónicas a expandirse hacia la TV por cable (no satelital) en ciudades grandes y medianas desde enero de 2018 (y sin límites desde enero de 2019). Este sendero hacia la convergencia no resolvió la disputa entre Telefónica y Clarín, que se acentuó a partir de la unión Telecom-Cablevisión. La fusionada es la única en condiciones de brindar servicios convergentes a lo largo y ancho del país. Si bien la capilaridad de la red del grupo español ofrece una posición estratégica para competir, la precariedad del tendido de fibra final sólo permite hacerlo en internet y la obliga a encarar fuertes inversiones para brindar TV por cable, lo cual se topa con su alto endeudamiento global y la inestabilidad del marco regulatorio (Fontanals, 2018). Por dicho motivo, las telcos reclaman vía libre para ofrecer televisión satelital. El sentido general de la re-regulación permite el libre desenvolvimiento de una lógica que tiende hacia la concentración convergente entre las telecomunicaciones y el audiovisual (Becerra y Mastrini, 2017). La justificación recayó en el diagnóstico de un mercado e infraestructura atrasados cuyo desarrollo requería la atracción de inversiones (Becerra y Mastrini, 2018).

A este panorama se suma la propagación de la oferta OTT que brinda contenidos audiovisuales a través de internet (Marino, 2018). Con firmas globales como Netflix que amenazan la distribución tradicional de TV, los actores clásicos desarrollan plataformas similares: Flow de Cablevisión-Telecom, Movistar TV de Telefónica y Claro TV de Telmex. 


\section{Zero-rating argentino}

En 2014, el Congreso sancionó la Ley 27078 Argentina Digital, cuyos artículos 56 y 57, aún vigentes tras las modificaciones del Decreto 267/2015, garantizan la neutralidad de la red de manera ex ante. A diferencia de otros países donde se regula ex post y los controlantes analizan caso por caso, como en Estados Unidos, el inciso b del 57 prohíbe expresamente la tasa cero, denegando a los prestadores "fijar el precio de acceso (...) en virtud de los contenidos, servicios, protocolos o aplicaciones (...) utilizados u ofrecidos a través de los respectivos contratos".

A pesar de ello, las operadoras móviles ofrecen promociones de zero-rating. Personal Telecom- dio el puntapié inicial en 2016 bonificando el envío de mensajes de texto, notas de voz, fotos y videos -excluyendo las llamadas y video llamadas-, de la aplicación de mensajería Whatsapp. Al año siguiente la imitaron Claro -América Móvil-, Movistar y la operadora virtual Tuenti -ambas de Telefónica-, para luego ampliar paulatinamente los destinatarios hasta cubrir tanto a los clientes prepagos como pospagos.

Esta estrategia es costeada por las telefónicas con los objetivos de captar y conservar clientes y de aumentar el consumo de datos (Califano, 2018), en un marco donde los precios de la telefonía móvil, a diferencia de la fija, no están regulados (Becerra y Mastrini, 2017). En el tercer trimestre de 2018, los accesos móviles habían ascendido 25\% respecto del mismo trimestre de 2015, mientras los fijos sólo 9\% (1). En los primeros tres trimestres de 2018, el consumo de datos de banda ancha móvil creció 59\% interanual y 503\% respecto del mismo período de 2015 (2). En ese marco, Whatsapp es utilizado por más del 90\% de los usuarios móviles de la Argentina -escoltado por Facebook Messenger, de la misma compañía, con un $60 \%$ - y representa el 5,6\% del consumo global de datos en Movistar (Catalano, 2017). Esta práctica no sólo incrementó el uso del mensajero, consultado al menos una vez por hora por el $74 \%$ de los usuarios de smartphones (Deloitte, 2017), sino que implica un obstáculo para otras aplicaciones existentes, como Telegram o WeChat, crea una barrera de entrada para las nuevas, afectando la competencia y la innovación, y supone el riesgo de convertirse en un jardín vallado.

Sumado a ello, Personal adoptó durante 2018 una segunda oferta de tasa cero al bonificar a los abonados que compartiera con Cablevisión la visualización de los partidos del Mundial de fútbol, de la final de la Copa Libertadores y del recital Personal Fest a través de su aplicación de video a demanda Flow. Esta modalidad supone que una operadora integrada verticalmente priorice no sólo una aplicación propia sino también contenidos producidos por y cuyos derechos de televisación pertenecen a socios comerciales. 
La producción de los partidos de Mundial de fútbol 2018 en la Argentina y los de la Copa Libertadores -hasta la edición 2018 inclusive- correspondió a la productora Torneos -cuyo principal accionista es DirecTV-. En tanto, los derechos de televisación del certamen de la FIFA se repartieron entre TyC Sports -una señal controlada en partes iguales por Torneos y el Grupo Clarín-, la TV Pública y DirecTV Sports. Por su parte, los derechos de transmisión de la competición continental corren por parte de Fox Sports Latinoamérica. Finalmente, tras sólo haberlo auspiciado durante más de una década, desde este año Personal se involucra de lleno en la producción del Personal Fest.

Durante el Mundial, la utilización de Flow creció un 20\% totalizando 13 millones de visualizaciones. En las dos primeras fechas, el $77 \%$ de sus usuarios acumularon 6,5 millones de visualizaciones, de las cuales el $35 \%$ provinieron desde celulares. Durante todo el evento, la promoción de zero rating impactó a 175 mil clientes de Personal. Al tercer trimestre de 2018, el servicio contaba con 1,2 millones de abonados -53\% más que a fines de 2017-, de los cuales el $81 \%$ utilizaba los smartphones para acceder a contenidos.

Al impulsar una aplicación propia, que aspira a convertirse en un servicio OTT, dicha estrategia implica como contrapartida un menoscabo de la posición de otros servicios de streaming audiovisual con los cuales compite, ya sea en la transmisión en vivo de señales puntuales, como Fox Play, en el consumo diferido de ficciones y documentales, como Netflix, o en un servicio de grilla que integre ambas ofertas, como Movistar TV. Al mismo tiempo, tiende a favorecer la captación y conservación de clientes tanto de Personal como de Cablevisión, así como la migración de estos últimos hacia los abonos más caros. No es la primera vez que el Grupo Clarín aprovecha contenidos de interés relevante, específicamente las transmisiones de partidos de fútbol, para crear ventajas por sobre sus competidores (Pérez, 2018).

\section{Zero-regulación}

ENACOM no adoptó hasta la fecha alguna resolución firme que vete dichas prácticas o las condicione, a pesar de haber incluido la protección de la neutralidad de la red en los Principios que regirán la Ley de Comunicaciones Convergentes. Al contrario, su par chileno dispuso una reglamentación específica sobre zero-rating (Fontanals, 2017b) y, al igual que el peruano, habilitó la estrategia a condición de empaquetarse con datos activos para acceder al resto de los contenidos (Fontanals, 2017a).

En el único expediente en la materia, Telefónica denunció a Telecom aduciendo que la bonificación de Flow durante el Mundial de fútbol violaba la neutralidad, entre otras 
disposiciones (3). La defensa lo rechazó, esgrimió que "se estaría eliminando, temporariamente, una restricción al liberarse el consumo de datos", achacó que aquélla obraba de manera idéntica con Whatsapp y la invitó a aplicar la misma estrategia con Movistar TV. Más de dos meses después y con el evento deportivo finalizado, la dirección de Desarrollo de la Competencia en Redes y Servicios de ENACOM concluyó que la promoción era tolerable siempre y cuando se aplicara también al resto de las aplicaciones similares, que en ese caso correspondía a aquellas a través de las cuales se hubiera podido visualizar dichos partidos. Al cierre de este artículo, el organismo mantiene el expediente abierto, aún no sancionó a Telecom y tampoco acusó recibo de la denuncia cruzada por bonificación de Whatsapp (4). En noviembre, y a raíz de que la fusionada repitió su estrategia para la final de la Copa Libertadores, Telefónica reiteró en dos oportunidades el reclamo. Finalmente, en diciembre Telecom dio marcha atrás con la promoción que había anunciado previamente respecto del Mundial de Clubes.

La postura de ente, revelada en dichos silencios que asumen la forma de una política no oficial (Freedman, 2015), es coherente con la orientación de sus iniciativas mencionada previamente. La mutación de la intervención gubernamental se inscribe en un nuevo paradigma regulatorio con una perspectiva más economicista que centrada en el bienestar social, más permeable a los intereses de las corporaciones, apostando por un regulador independiente e imparcial y a la aplicación ex post de reglas generales de defensa de la competencia entre privados más que a aquellas específicas de protección de la diversidad cultural (Freedman, 2006; Cuilenburg y McQuail, 2003; Mastrini, De Charras y Fariña, 2011; Rodríguez Miranda y Carboni, 2012). Sin embargo, lejos de cumplimentar los dos últimos componentes, aquí la connivencia entre reguladores y regulados derivó en una adecuación errática y paulatina de las normas a los intereses y necesidades expansivas de uno los agentes (Freedman, 2015; Becerra, 2018) y cuya máxima expresión la constituye la doblemente aprobada fusión consignada. En el marco de un mercado infocomunicacional cada vez más globalizado, dicha estrategia, denunciada por los otros competidores concentrados de capitales extranjeros, se comprende como una apuesta a conformar un "campeón nacional" que actúe como barrera para aquéllos y cuente con posibilidades de expandirse en la región (Becerra y Mastrini, 2017).

\section{Conclusiones}

La protección de la neutralidad de la red es necesaria en el contexto convergente para limitar la tendencia del mercado infocomunicacional a la concentración y resguardar el derecho a la 
información de la población. En tanto terreno de disputa entre actores dominantes es conveniente que no se restrinja a los operadores de red y se considere extenderla a las plataformas. Allí, donde se despliegan jugadores globalizados que escapan a la territorialidad del Estado nación, el desafío es cómo evitar que la regulación se delegue a intereses privados. Aunque haya sido especificado en la normativa, el zero-rating es una de las violaciones a la neutralidad más toleradas alrededor del mundo. En la Argentina, las prestadoras de internet móvil costean servicios ajenos para posicionar su oferta, mientras una de ellas, integrada verticalmente, también favorece sus propios contenidos. Consistentemente con la reorientación de las políticas de comunicación en favor de los actores concentrados tras el cambio de gobierno, y en particular del mayor de ellos, el regulador ni independiente ni imparcial no lo prohíbe ni condiciona. Su única actuación, aún sin sanciones, fue consecuencia de una gresca entre aquéllos.

Notas

(1) INDEC. Encuesta a Proveedores del Servicio de Acceso a Internet. Recuperado de https://bit.ly/2L8XKaF

(2) INDEC. Encuesta mensual de telefonía fija y móvil. Recuperado de https://bit.ly/2UM2JNQ

(3) La denuncia, la defensa y las actuaciones de ENACOM forman parte del expediente EX-2018-26255540-APNAMEYS\#ENACOM, consultado el 14 de diciembre de 2018.

(4) Ante el pedido de acceso a la información pública requiriendo actuaciones o documentos donde se abordaran casos o problemáticas de tasa cero, la respuesta de ENACOM el 26 de noviembre de 2018 en IF-2018-61159345-APNSD\#ENACOM únicamente dio cuenta de la denuncia aquí presentada.

\section{Bibliografía}

Becerra, M. (2003). De la divergencia a la convergencia (Capítulo 6). En Sociedad de la información: proyecto, convergencia, divergencia. Buenos Aires: Grupo Editorial Norma.

Becerra, M. (2015). Argentina: tres décadas de políticas de comunicación en democracia y Medios políticas y redes: la revolución inconclusa. En De la concentración a la convergencia. Políticas de medios en Argentina y América Latina. Buenos Aires: Paidós. 
Becerra, M. y Mastrini, G. (2017). Economía de la comunicación y la cultura (Capítulo 1). En La concentración infocomunicacional en América Latina en el siglo XXI (2000-2015) (pp. 21-62). Bernal: Universidad Nacional de Quilmes.

Becerra, M. (2017). Intereses de la neutralidad de la red. Revista Colsecor. Recuperado de http://colsecornoticias.com.ar/index.php/archivo/7990-intereses-de-la-neutralidad-de-lared

Becerra, M. (2018a). Adecuarse a la concentración extrema. Letra P. Recuperado de https://www.letrap.com.ar/nota/2018-6-29-19-35-0-adecuarse-a-la-concentracionextrema

Becerra, M. (2018). Paradojas de la economía macrista para Clarín. Letra P. Recuperado de www.letrap.com.ar/nota/2018-8-22-16-47-0-paradojas-de-la-economia-macrista-paraclarin

Becerra, M. y Mastrini, G. (2018). Más dueños que nunca. Revista Anfibia. Recuperado de http://revistaanfibia.com/ensayo/mas-duenos-nunca

Califano, B. (2018). Los límites del "WhatsApp ilimitado". Revista Fibra, 20, mayo.

Califano, B. y Baladrón, M. (2013). La regulación de Internet: formas emergentes de gobierno privado. En Mastrini, G.; Bizberge, A. y De Charras, D. (Eds.). Las políticas de comunicación en el siglo XXI. Nuevos y viejos desafíos (pp. 207-242). Buenos Aires: La Crujía.

Chaparro, E. (2014). Neutralidad de la red: indefiniciones e imprecisiones a la orden del día. Fundación Vía Libre, Buenos Aires. Recuperado de https://bit.ly/2J60P8u

Cisco (2018). Cisco Visual Networking Index: Forecast and Trends, 2017-2022. Recuperado de https://bit.ly/2GPqVeA

Comscore (2017). Mobile Global Report Comparativa internacional sobre tendencias y comportamientos móviles. Recuperado de https://www.amic.media/media/files/file_352_1408.pdf

Cortés Castillo, C. (2014). La neutralidad de la red: la tensión entre la no discriminación y la gestión y Vigilancia en la red: ¿qué significa monitorear y detectar contenidos en internet. En Bertoni, E. (comp.). Internet y derechos humanos. Aportes para la discusión en América Latina. Buenos Aires: CELE-Universidad de Palermo.

Cortés Castillo, C. (2016). La gobernanza de internet: la trampa de las formas. En Bertoni, E. A. (comp.). Internet y derechos humanos II: aportes para la discusión de políticas públicas en América (pp. 11-33). Ciudad Autónoma de Buenos Aires: Ediciones del Jinete Insomne. 
Cuilenburg, J. van y McQuail, D. (2003). Cambios en el paradigma de política de medios. Hacia un nuevo paradigma de políticas de comunicación. European Journal of Communication, 18(2), Sage, Londres, pp 181-207.

Deloitte (diciembre de 2017). Consumo móvil en Argentina Los móviles prueban ser indispensables en un mundo "siempre" conectado. Recuperado de https://bit.ly/2OW7jrf

Fernández, P. E. (2014). Neutralidad de la Red: tensiones para pensar la regulación de Internet. Question, 1, Facultad de Periodismo y Comunicación Social, La Plata.

Fontanals, G. (2016). La neutralidad de la red, la apertura de internet. Revista Fibra, 8, enero.

Fontanals, G. (2017a). La neutralidad de la red y el zero-rating. Revista Fibra, 14, abril.

Fontanals, G. (2017b). Las políticas de neutralidad de la red. Su legislación en el mundo y en América Latina. Revista Fibra, 16, julio.

Fontanals, G. (2017c). Pariendo a Goliat. Revista Fibra, 17, septiembre.

Fontanals, G. (2018). La convergencia como excusa. Revista Fibra, 18, enero.

Freedman, D. (2006). Las actuales dinámicas del poder en la elaboración de políticas de medios. Media, Culture \& Society, 28, 1-12.

Freedman, D. (2015). Media Policy Fetishism. Critical Studies in Media Communication, 32(2), 96-111. doi: http://doi.org/10.1080/15295036.2015.1024139

Gendler, M. A. (2017). La Neutralidad de la Red: Debates y acciones en Argentina. XXXI Congreso ALAS Uruguay 2017.

Intervozes y Derechos Digitales (2017). Neutralidad de red en América Latina: reglamentación, aplicación de la ley y perspectivas. Los casos de Chile, Colombia, Brasil y México. San Pablo (Brasil): Intervozes; Santiago (Chile): Derechos Digitales.

Marino, S. (2018a). Políticas para el Espacio Audiovisual Ampliado de Cambiemos: ¿falsas promesas? Revista Fibra, 18, enero.

Marino, S. (2018b). La oferta del audiovisual ¿ampliada? Revista Fibra, 20, mayo.

Mastrini, G.; De Charras, D. y Fariña, C. (2011). Nuevas formas de regulación internacional y su impacto en el ámbito latinoamericano. Revista de Economía Política de las Tecnologías de la Información y Comunicación, XIII(3), 1-30.

McSherry C.; Malcolm, J. y Walsh K (2016). Tasa Cero (Zero Rating): Qué es y por qué debería importarte. Electronic Frontier Foundation. Recuperado de https://bit.ly/2GN2IFD

Observacom (2018). Viejos y nuevos gatekeepers en Internet. Recuperado de https://bit.ly/2VKOOUO

Pallero, J. (2018). Neutralidad de la red: ¿qué te puedo cobrar? El Gato y la caja. Recuperado de https://elgatoylacaja.com.ar/neutralidad-de-la-red-que-te-puedo-cobrar/

Pérez, G. (2018). Crónica de una fusión anunciada. Revista Fibra, 17, septiembre. 
Rodríguez Miranda, C. y Carboni, O. (2012). Neutralidad de la red, un debate pendiente en Argentina. Oficios Terrestres, 1(28). Recuperado de https://bit.ly/2XRN01A

Sandvine (2018). The Global Internet Phenomena Report October 2018. Recuperado de www.sandvine.com/hubfs/downloads/phenomena/2018-phenomena-report.pdf

Sumits A. (28 de agosto de 2015). The History and Future of Internet Traffic. Cisco Blogs. Recuperado de https://blogs.cisco.com/sp/the-history-and-future-of-internet-traffic 